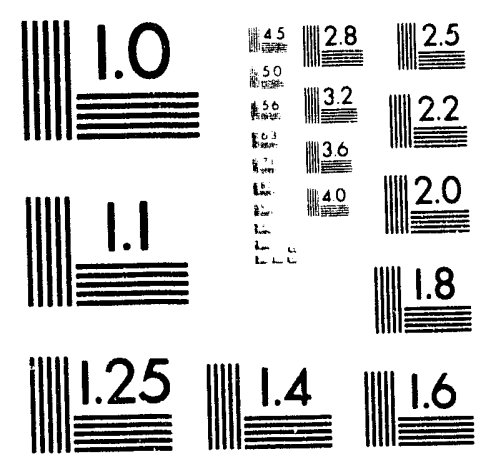



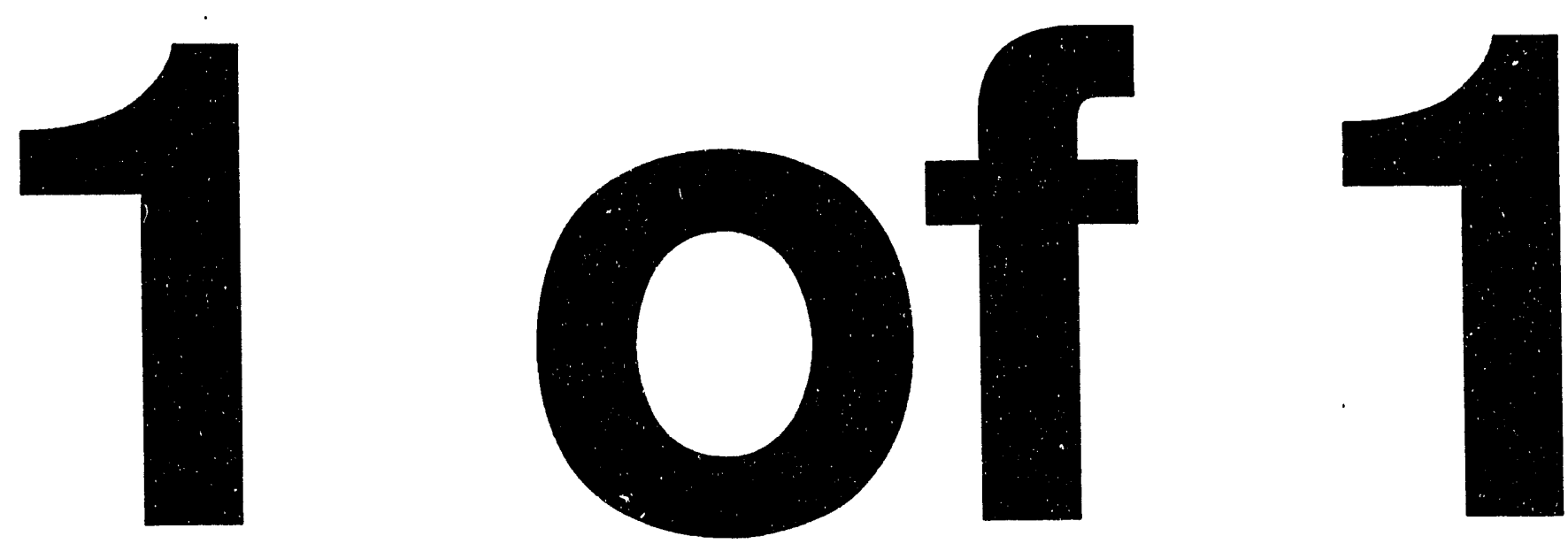
This report was prepared as an account of work sponsored by an agency of the United States Government. Neither the United States Guvernment nor any agency thereof, nor any of their employees, makes any warranty, express or implied, or assumes any legal liability or responsibility for the accuracy, completeness, or usefulness of any information, apparatus, product, or process disclosed, or represents that its use would not infringe privately owned rights. Reference herein to any specific commercial product, process, or service by trade name, trademark, manufacturer, or otherwise does not necessarily constitute or imply its endorsement, recommendation, or favoring by the United States Government or any agency thereof. The views and opinions of authors expressed herein do not necessarily state or reflect those of the United States Government or any agency thereof.

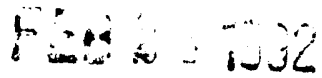

SNO-STR-91-60

\title{
EFFECT OF PANEL ALIGNMENT AND SURFACE FINISH ON BOND STRENGTH
}

\author{
Jan M. Wouters and Peter J. Doe \\ Les Alamos National Laboratory \\ Los Alamos, NM 87545 \\ William E. Baker \\ University of New Mexico \\ Albuquerque, NM
}

October, 1991

\begin{abstract}
The flexural strength of bonded acrylic is tested as a function of panel alignment and bond surface finish. Bond strength was shown to be highly dependent on both parameters with onlv a narrow range of values yielding a high strength bond.
\end{abstract}

\section{Introduction}

This report is one in a series which describe tests designed to evaluate the envelope of bond joints that will be acceptable in construction of the heavy water containing acrylic vessel for the Sudbury Neutrino Observatory (SNO) detector. In this report we address two questions: 1) what is the effect of misalignment and mismatched thickness on the strength of two sheets being bonded

\footnotetext{
This work was supported in part by the Department of Energy and by the Los Alamos
National Laboratory.
} 
together, and 2) for a given bond, how much finish work (and in some cases repair work) is needed to meet minimum criteria for the bond strength in the final acrylic vessel for SNO.

A portion of this bond envelope was mapped out in a previous report, SNO-STR-91-03, where the tensile strength versus bond joint thickness was evaluated. In the present report we examine flexural strength as a function of bond preparation: considering overfilling or underfilling of the joint with the adherive, mismatch in thickness of the acrylic sheets, and misalignment of the sheets. In addition, we finished the bond joint surface of each bond joint type in several different ways after the adhesive has polymerized.

\section{Experimental technique}

\section{General testing procedure}

Four different oint samples (Types: $A, B, C, D$ ) where prepared with different amounts of filling and varying amounts of misalignment of the two glued sheets. These samples where then subdivided, creating four variations for each type (Subtypes: 1, 2, $3,4)$ with differences in how the bond surface finishing was done. The various "types" and "subtypes" are listed in Table 1 with cross sections of different bond joints shown in Figure 1. The only common joint parameter between all samples was the use of a 1/8" initial thickness bond joint that we determined to be optimal in a previous study (see SNO-STR-91-3).

\begin{tabular}{|c|c|c|c|}
\hline & Sample type & $\begin{array}{c}\text { Sub } \\
\#\end{array}$ & Subtype \\
\hline \multirow[t]{4}{*}{$A$} & \multirow{4}{*}{$\begin{array}{l}\text { Two 1" thick pieces bonded } \\
\text { together. Bond is always } \\
\text { overfilled. Pieces are } \\
\text { aligned. }\end{array}$} & 1 & No finishing \\
\hline & & 2 & Bond is routed flush \\
\hline & & 3 & $2+$ bond is sanded \\
\hline & & 4 & $3+$ bond is polished \\
\hline \multirow[t]{2}{*}{$B$} & \multirow{2}{*}{$\begin{array}{l}\text { Two 1" thick pieces bonded } \\
\text { together. Bond is always } \\
\text { underfilled. Pieces are }\end{array}$} & 1 & No finishing \\
\hline & & 2 & $\begin{array}{l}\text { Bond is routed, sanded, and } \\
\text { polished }\end{array}$ \\
\hline
\end{tabular}




\begin{tabular}{|c|c|c|c|}
\hline & \multirow[t]{2}{*}{ aligned. } & \multirow{2}{*}{\begin{tabular}{|l|}
3 \\
4 \\
\end{tabular}} & $\begin{array}{l}\text { Bond is filled, routed, } \\
\text { sanded and polished }\end{array}$ \\
\hline & & & $\begin{array}{l}\text { Bond is flaired by sanding } \\
\text { and then polished. }\end{array}$ \\
\hline \multirow[t]{4}{*}{$C$} & \multirow{4}{*}{$\begin{array}{l}1 " \text { thick piece bonded to } \\
0.75 " \text { thick piece. Bond is } \\
\text { always filled. Pieces are } \\
\text { aligned. }\end{array}$} & 1 & No finishing \\
\hline & & 2 & Bond is routed to a taper \\
\hline & & 3 & $2+$ bond is sanded \\
\hline & & 4 & $3+$ bond is polished \\
\hline \multirow[t]{4}{*}{$\bar{D}$} & \multirow{4}{*}{$\begin{array}{l}\text { Two 1" thick pieces bonded } \\
\text { together. Bond is always } \\
\text { filled. Pieces are mis- } \\
\text { aligned }\end{array}$} & 1 & No finishing \\
\hline & & 2 & Bond is routed flush \\
\hline & & 3 & $2+$ bond is sanded \\
\hline & & 4 & $3+$ bond is polished \\
\hline
\end{tabular}

Table 1: Summary of 4 bond types (A-D) and variations on each type $(1-4)$.

Figure 1 Schematic of sample types and subtypes.

Bond Finish for 4 Sub Samples

(Type A - Overfilled joint)
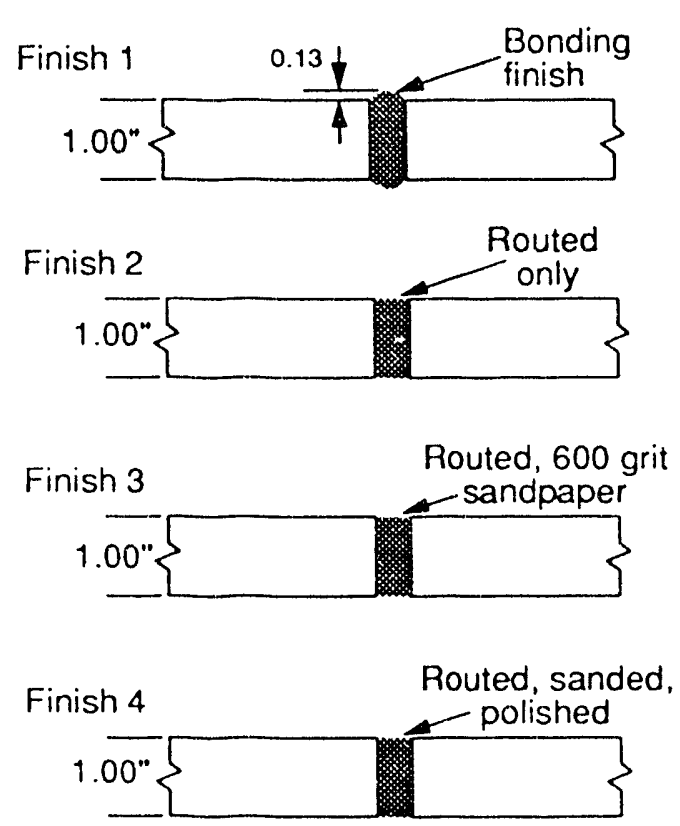

Bond Finish for 4 Sub Samples

(Type E3 - Underfilled joints)

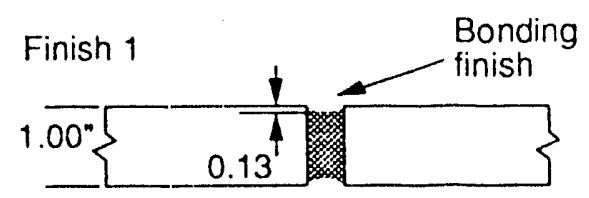

Finish 2 Routed, sanded,

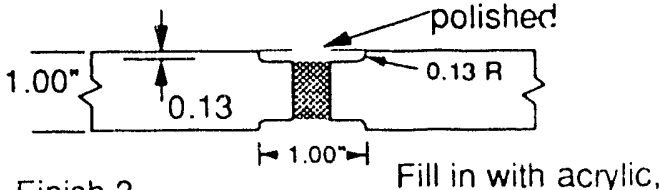

$\begin{array}{cc}\text { Finish } 3 & \text { Fill in with acrylic, } \\ \text { Route, sand, polish }\end{array}$

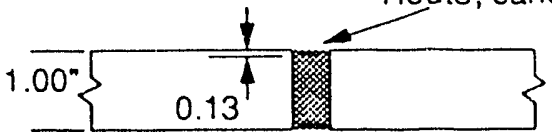

Finish 4 Flair in by sanding,

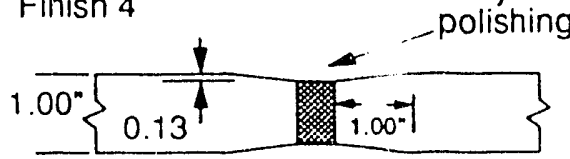



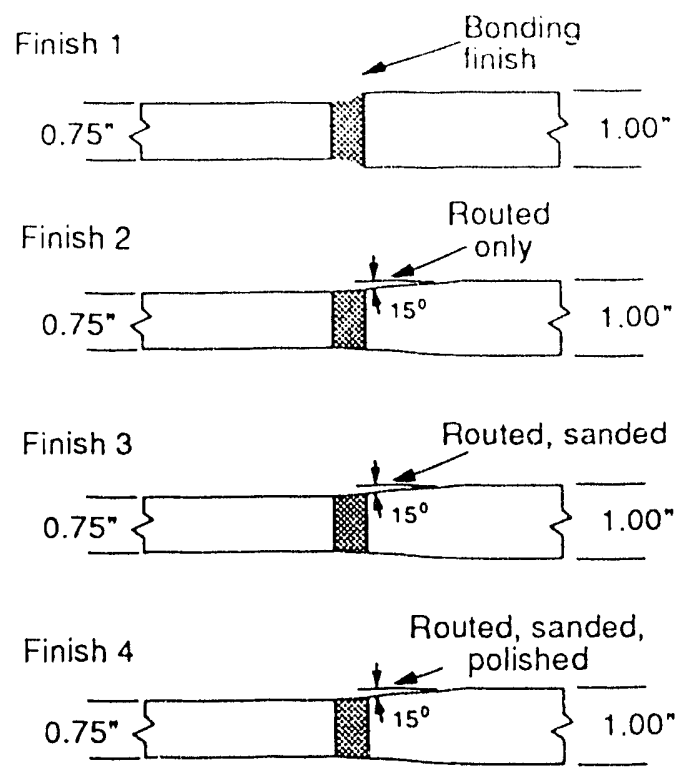
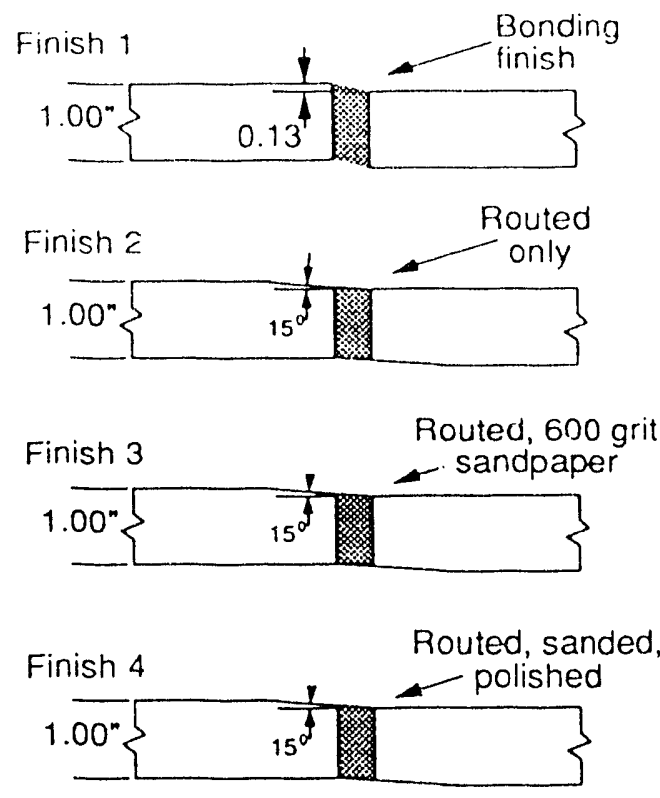

Each of the bond types was prepared by gluing together two 20" wide by 10 "long by 1 " thick (one piece was 0.75 " for type C) pieces of acrylic along the 20" edge. The resulting large sheets where then divided into four equal size samples. Each $1 / 4$ sheet was finished differently into the various subtypes and then further subdivided into 6 individual test samples approximately $1 " \times 1 " \times 20$ ". These samples were placed in a four point flexural testing fixture and loaded to failure

\section{Sample preparation}

All samples were prepared according to the specifications listed in ASTM D790-86 for the flexural testing of acrylic. The material used was ultra violet transmitting cast acrylic sheet (ROHAGLAS GS218 UVT acrylic) purchased from Rohm - Germany, a large supplier of scientific grade acrylic. Eight rectangular pieces measuring 29" wide, 10" long, and 1" thick were cut from a single sheet of acrylic and all edges machined to a 63 finish. The top and bottom optical quality surfaces were not disturbed. 
For sample type $C$ the top surface of one piece was machined (63 finish) so that the final thickness was 0.75 ".

These rectangular panels were then sent for bonding to Reynolds Polymer Technology (RPT) in Irvine, California. This company has extensive experience in the casting, thermoforming, polishing and bonding of very large acrylic panels. They were asked to use their best proprietary bonding procedure for joining our panels. This procedure begins with the annealing of all panels because all SN.JO vessel panels will be pre-annealed. Next the two bonding surfaces (the 29.0" long sides of the panels) are sanded using 120 grit sandpaper. The panels are then aligned and spaced 1/8" apart. All joints are taped to contain the bonding compound and the shims are removed prior to bonding to allow the panels to "relax" while the bond cures. This relaxation is important since the bonding compound shrinks by several percent and we wanted to minimize the stresses on the bond joints.

In this test, RPT used their proprietary formulation of adhesive based on methyl methacrylate, the acrylic monomer (lot PMI6632), for bonding the panels. Once the bonding compound is applied the samples are cured at temperatures up to $85 .{ }^{\circ} \mathrm{C}$. Finally the samples are probed with a fine wire to assure a good cure and the tape is removed. The bonded samples were not annealed since such annealing will not be possible during the construction of the acrylic vessel.

The 4 finished bonded panels where then cut using a table saw into four equal length subpanels each now measuring 6.9" wide, 20" long, and 1" thick. The finishing operations to produce the various subtypes listed in table 1 included one or more of the following procedures: routing, sanding, and polishing done to the top and bottom surfaces. The sides were not touched. For all routing operations a standard hand router was used. 60 to 120 grit sandpaper was then ised for those samples that were just sanded. For the polished samples sanding was continued until 400 
grit sandpaper was used. Polishing itself was done in several steps beginning with 1 micron alumina and continuing through 0.3 micron alumina.

These subpanels were then further subdivided using a band saw into pieces measuring approximately 1" wide, 20" long, and 1" thick. At this point the sides were finished using a milling machine to get a good machined edge free of surface scratches. A carbide milling tool was used with cuts limited to 0.1 " and finish cuts kept below 0.01". Only water was used as a lubricant. The top and bottom surfaces now prepared according to the procedures listed in Table 1 were not disturbed.

\section{Sample testing}

The specimens were tested in accordance with ASTM specifications D790-86, test method II, to determine the flexural strength of the bond joints. The testing was done at the University of New Mexico on a 20,000 lbs capacity Instron testing machine. A special four-point loading fixture was designed and fabricated to satisfy the ASTM testing specifications. A photograph of this fixture with a simulated specimen in place is shown in Figure $2 a$ and a schematic is shown in Figure $2 b$. The cylindrical loading noses have a rotational degree of freedom to minimze any torsion introduced in the specimen from non-parallel surfaces on the specimen. 
Figure 2a: Photograph of 4 point test fixture.

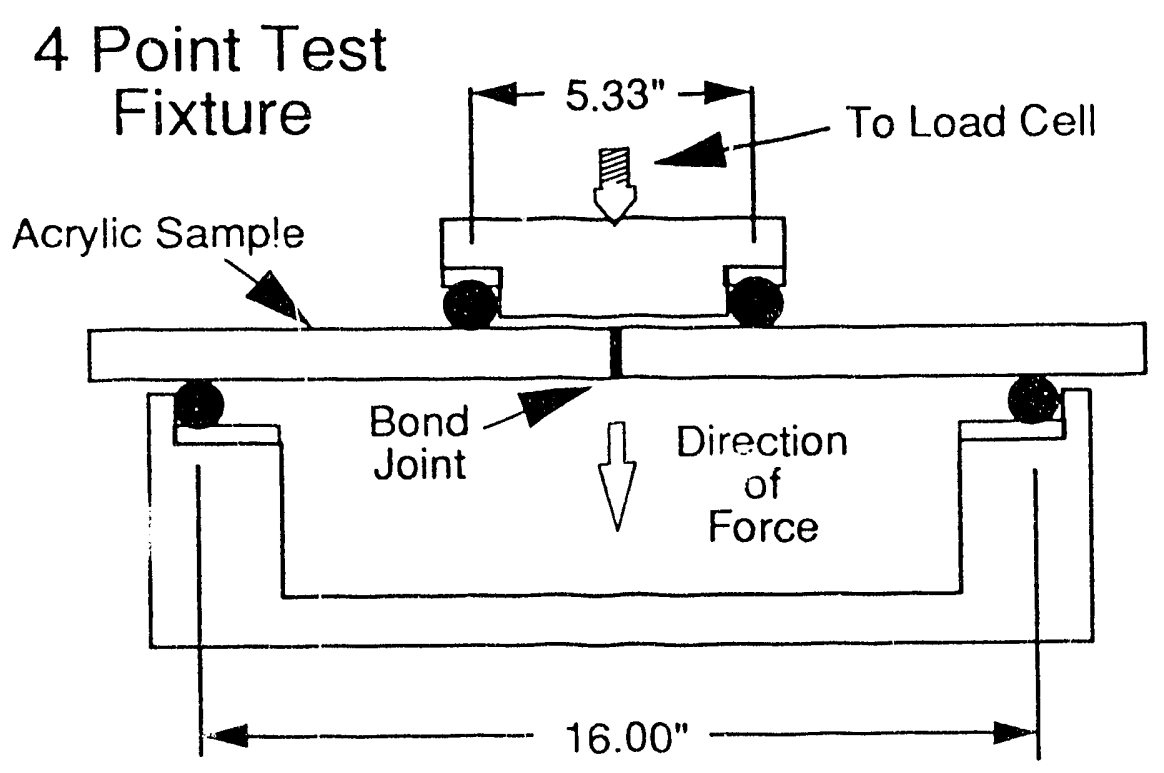

Figure 2b: Diagram of Flexural Testing Jig.

The applied force was measured with strain gauge load cells of $500 \mathrm{lbs}$ and $5000 \mathrm{lbs}$ capacity. The sensitivities of the load cells are traceable to NIST standards. The output of the load cells during a test was measured with a recording digital voltmeter, with the peak reading being used to calculate the failure load. 
Computation of the flexural strength was done using the equation:

$$
S=\frac{P L}{b d^{2}}
$$

where:

$S=$ flexural strength of the beam (psi)

$P=$ load at failure of the beam applied to the top of the load fixture (lbs).

$\mathrm{L}=$ distance between the outer load supports (in).

$b=$ width of the beam (in).

$d=$ depth of the beam (in).

This equation is from mechanics of material and is based upon the assumption that the material is linear to fracture.

Prior to testing, measurements of $b$ and $d$ were made. The depth was measured on each side of the bond joint, and the width was measured near the center. In reducing the data, the average of the two depth measurements was used for beams fabricated to have the same depth at each end. For beams having one end which was only 0.75 " deep by design, the depth used in the data reduction was for a 1" nominal end. This procedure was used to obtain an "effective strength of the material/joint configuration, i.e. a numerical value which could be meaningfully compared to strength values for specimens which were 1" thick on both ends. Stated differently, the goal was to compare the flexural strength for each sample to that of an idealized joint that has the full cross sectional area. Thus, even though the actual flexural strength of the material (or bond) may be similar in all specimens, the effective strength in specimens which have been routed to reduced thickness would be substantially less and endanger the integrity of the acrylic vessel. Our cross sectional measurements had the sole purpose of making minor coriections 'etween parent material dimensions, but not to correct for changes in bond dimensions. 


\section{Results}

Figures 3-6 show the raw results for bond finish types $A-D$. Figure 7 shows the average result for each type of bonded surface along with 1 sigma error bars. Figure 8 is identical to Fig. 7 except that the individual test results that deviate by more than 2 sigma from the average have been removed.

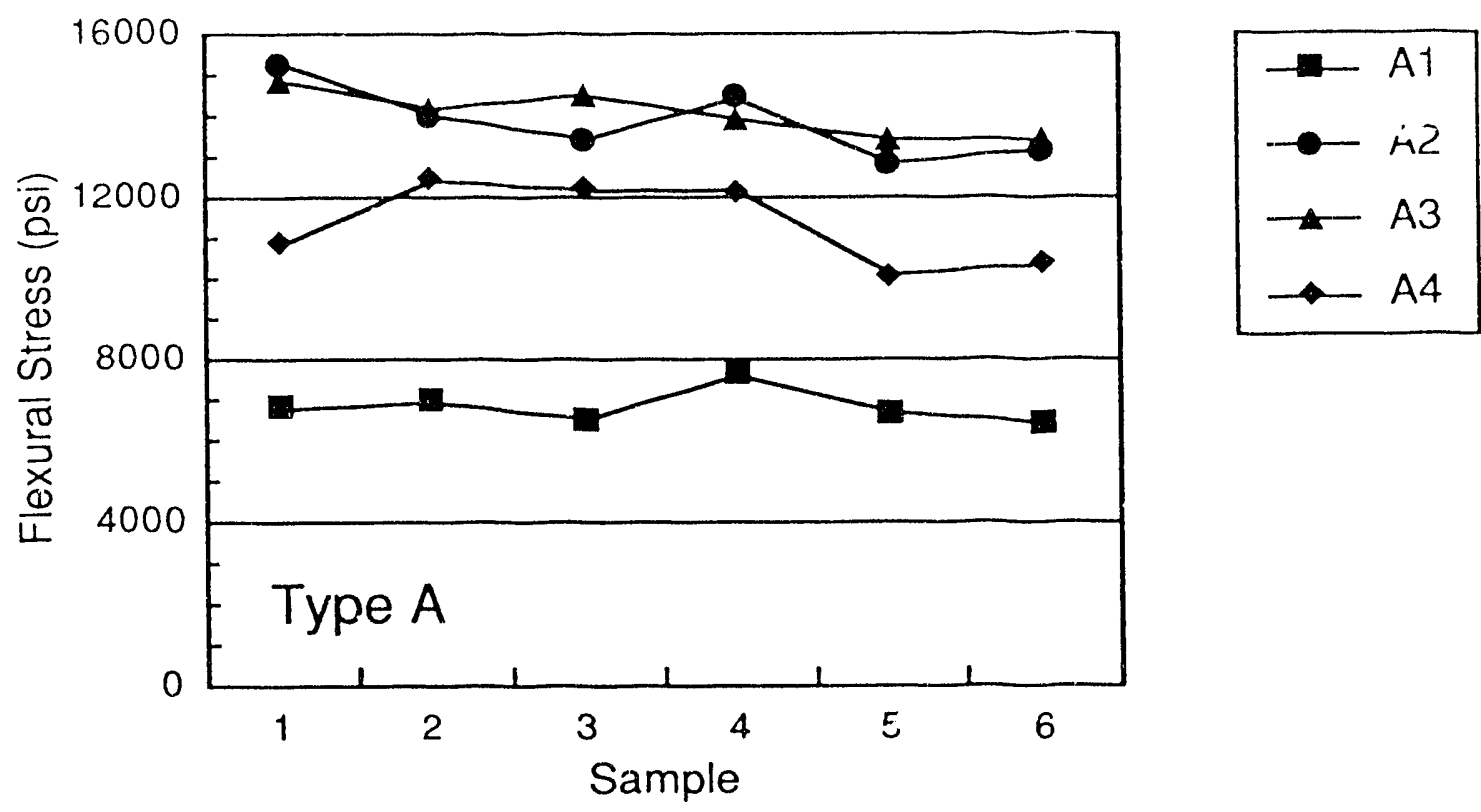

Figure 3: Flexural test samples A - overfilled joint 


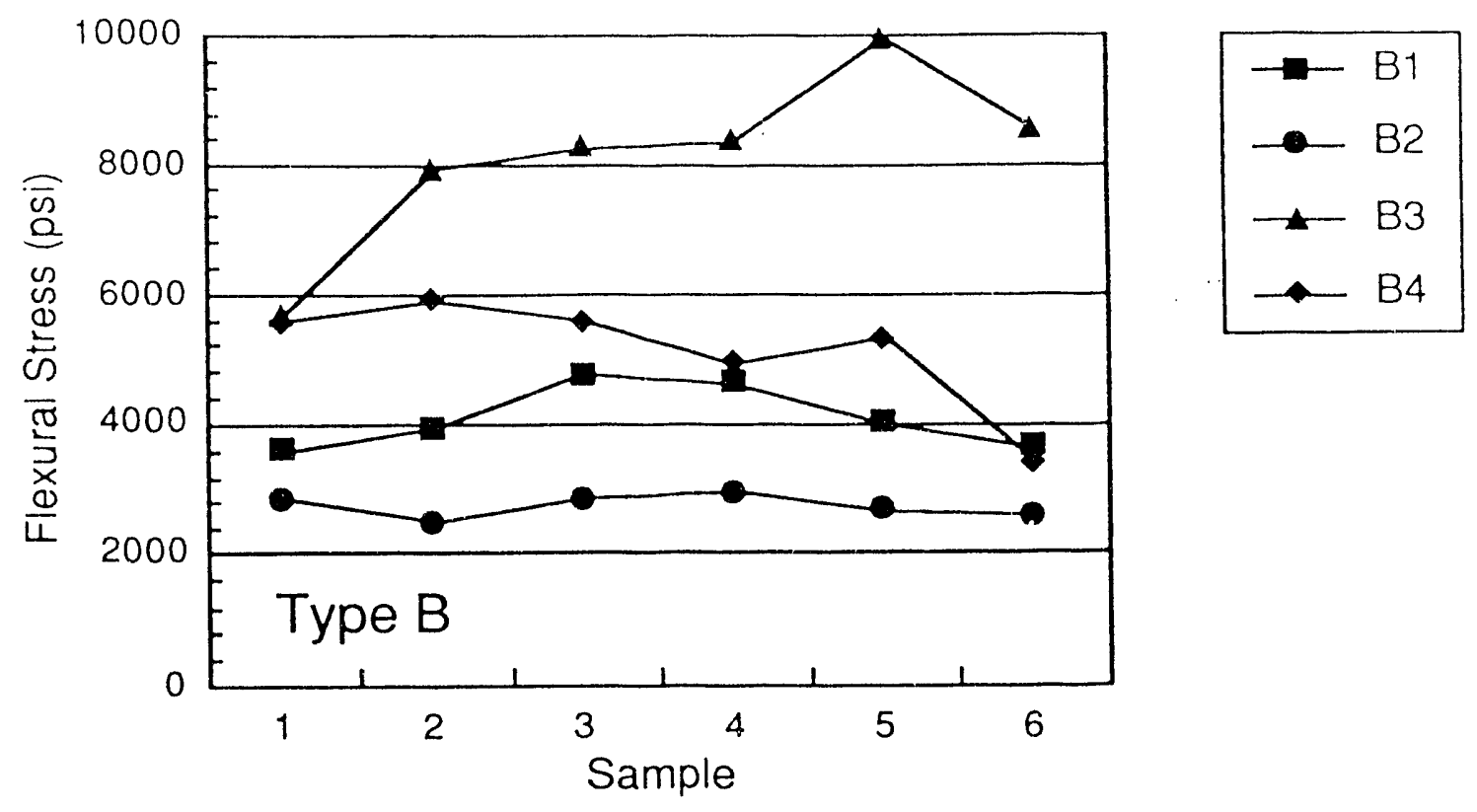

Figure 4: Flexural test samples A - underfilled joint.
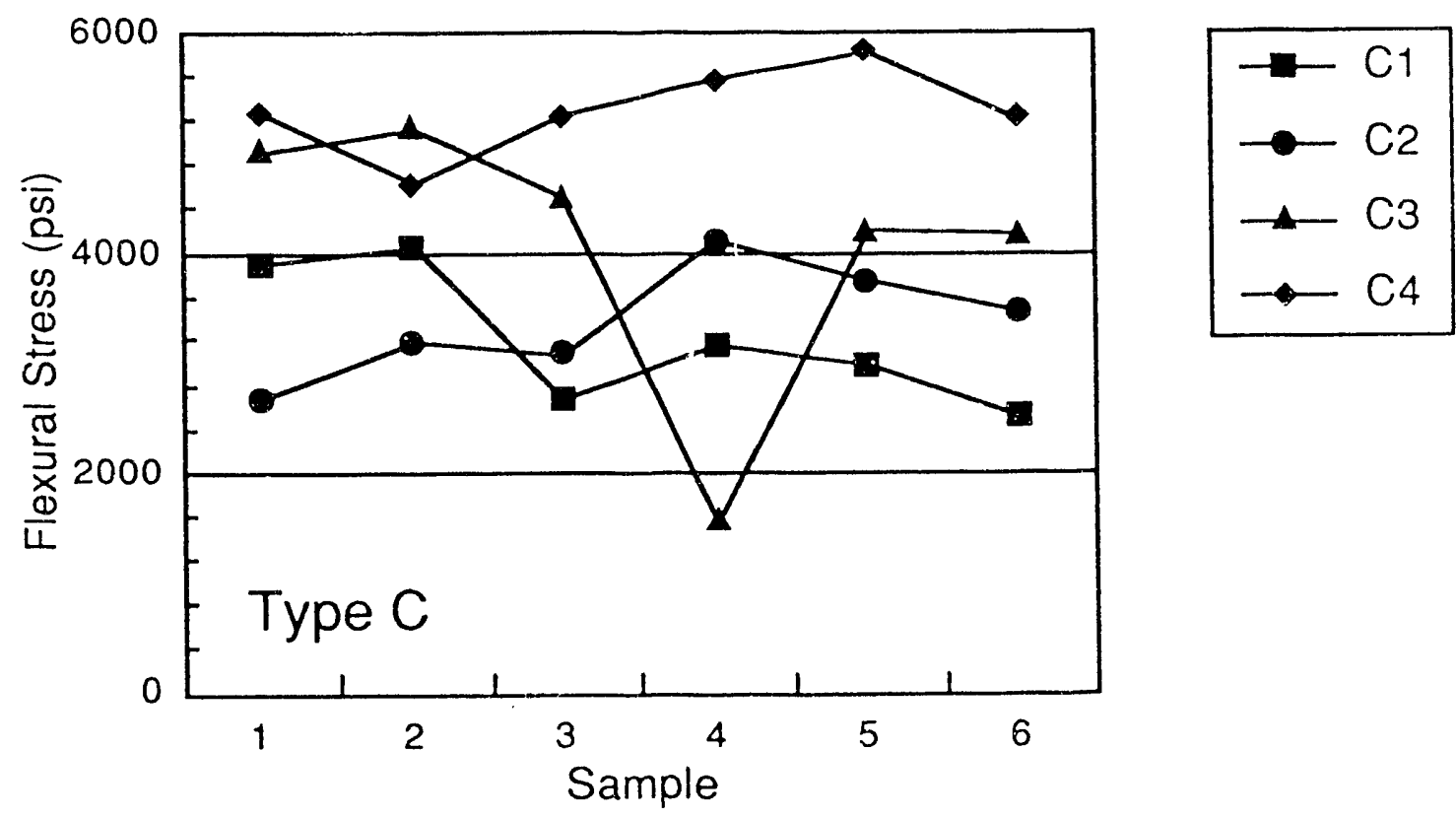

Figure 5: Flexural test samples C - one side only 0.75 " thick. 


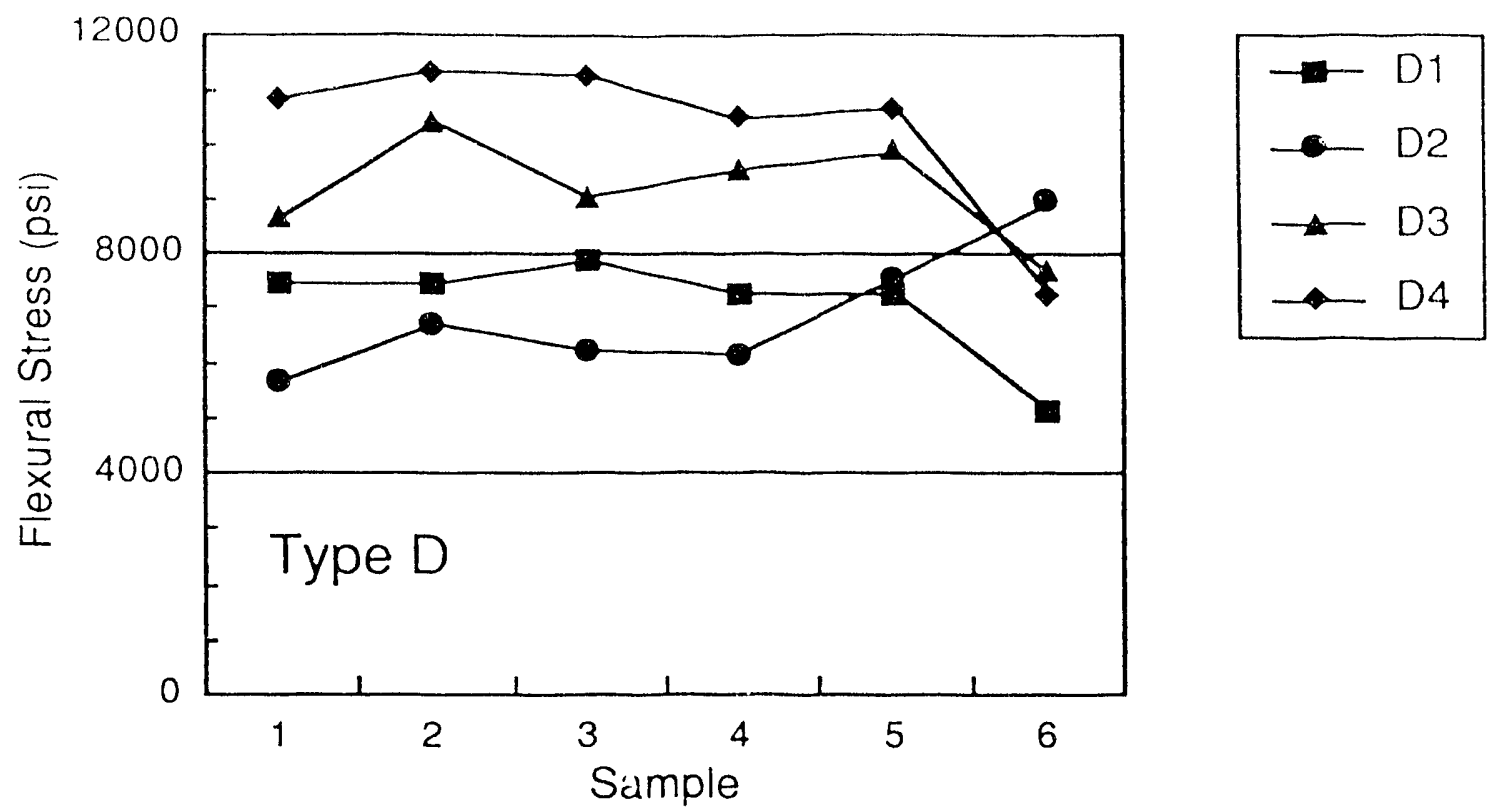

Figure 6: Flexural test samples D - misaligned acrylic.

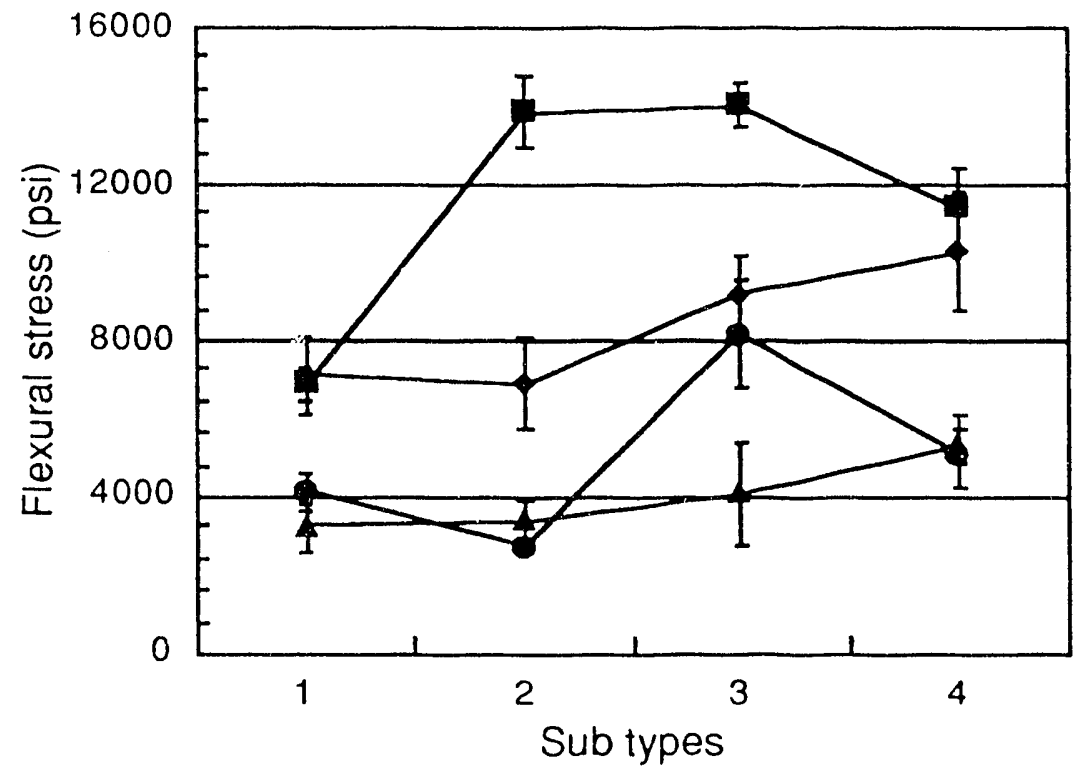

$$
\begin{aligned}
& \rightarrow \text { A Type } \\
& - \text { B Type } \\
& - \text { O Type } \\
& - \text { D Type }
\end{aligned}
$$

Figure 7: Average results for all samples 


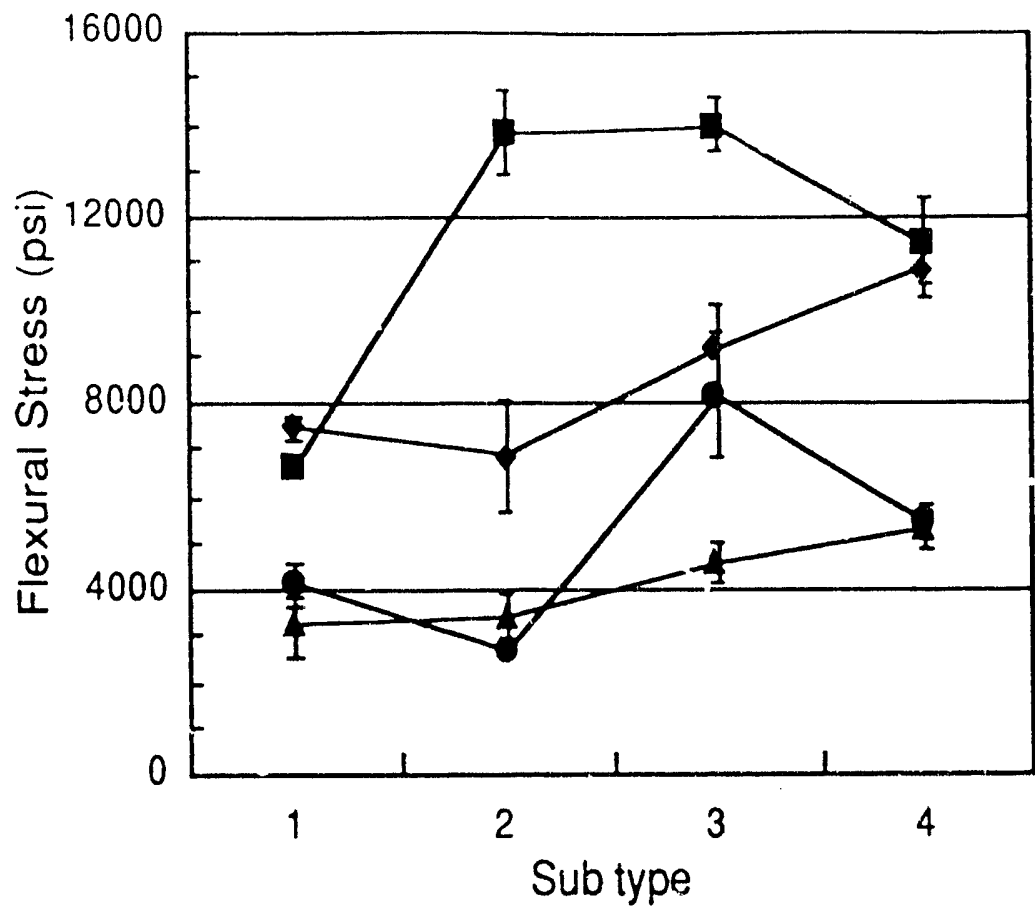

$$
\begin{aligned}
& \rightarrow \text { A Type } \\
& \rightarrow \text { B Type } \\
& \rightarrow \text { C Type } \\
& \rightarrow \text { D Type }
\end{aligned}
$$

Figure 8: Corrected average results for all samples

Table 2 lists the sample type, the flexural failure stress, and the standard deviation in this stress. This table also lists a revised failure stress and standard deviation in those cases where a single measurement is more than two standard deviations away from the mean. The standard deviation is calculated as follows:

STD DEV $=\sqrt{\frac{\left(X-X_{\text {ave }}\right)^{2}}{N-1}}$ where $X$ is the flexural failure stress

\begin{tabular}{|c|c|c|c|c|c|c|c|}
\hline & $\begin{array}{r}\text { Flexural } \\
\text { Stress } \\
\text { psi }\end{array}$ & $\begin{array}{l}\text { Failure } \\
\text { dev } \\
\text { psi }\end{array}$ & $\begin{array}{c}\text { Stress } \\
\text { Stress } \\
\text { (dev) }\end{array}$ & & $\begin{array}{c}\text { Flexural } \\
\text { Stress } \\
\text { psi }\end{array}$ & $\begin{array}{l}\text { Failure } \\
\text { dev } \\
\text { psi }\end{array}$ & $\begin{array}{c}\text { Stress } \\
\text { Stress } \\
\text { (dev) }\end{array}$ \\
\hline A 1 & 6827 & 420 & $6672(204)$ & C1 & 3200 & 635 & \\
\hline$A 2$ & 13822 & 901 & & $\mathrm{C} 2$ & 3371 & 517 & \\
\hline A 3 & 10999 & 580 & & C3 & 4066 & 1297 & $4571(442)$ \\
\hline A 4 & 11350 & 1049 & & $\mathrm{C} 4$ & 5289 & 395 & \\
\hline
\end{tabular}
and $N$ is the number of test coupons ( 6 in all our tests). Note that for these measurements the systematic errors in the testing procedure are considered to be negligible. 


\begin{tabular}{l|rrl|r|rrr|} 
B1 & 4084 & 488 & & D1 & 7051 & 996 & $7447(257)$ \\
B2 & 2733 & 177 & & D2 & 6862 & 1202 & \\
B3 & 8124 & 1371 & & D3 & 9186 & 959 & \\
B4 & 5114 & 898 & $5545(368)$ & D4 & 10291 & 1517 & $10896(359)$ \\
\hline
\end{tabular}

Table 2: Summary of acrylic flexural strength tests

\section{Discussion}

In this sectinn we describe, in turn, the test results for each iype of bond finich. What will become apparent by the end of the last type of bonc finish is that several clear conclusions can be made concerning the bond parameters and bond surface finish if one is striving to maximize the strength of these joints. The most important point to note from Table 2 is the flexural strength of a bond joint decreases very quickly with the presence of any imperfections so the envelope of allowable bond joint parameters and finishes is quite small.

A general comment that can be made about the failure of all the samples is that a high flexural strength results in the breakage surfaces being very jagged, while a low flexural strength created surfaces that looked as if that had been cut with a hot knife. This observation is almost certainly due to the different amounts of stored energy in the failure region at the moment of failure. Indeed, in many of the high flexural strength samples portions of the acrylic from the region of failure were ejected from the side of the sample under compression.

\section{A Samples}

For the overfilled bond joint, which is the "standard" bond joint produced by RPT, the results shown in Figure 3 support the first observation that surface finish is important to bond flexural strength. For the $A 1$ samples (slightly overfilled bond joint) the flexural strength of the joints is half of the best $A$ type samples. A close examination of the $A 1$ test coupons shows that the 
failure of every sample occured to one side of the joint in the parent material. (The joint itself was usually $20 \%$ thicker than the parent material.) However, the location of the failure was enevitably associated with a surface imperfection that we attribute to excess monomer from the joint spilling over the surface of the parent acrylic. This spill-over extends roughly $1 / 4$ " on either side of the joint centerline.

For the the $A 2$ (overfilled and routed) and $A 3$ (overfilled, routed and sanded) samples we see that the flexural strengths are quite comparable and high. These results demonstrate that just removing the gross surface imperfections vastly strengthens the joint. In most $A 2$ and $A 3$ joints the initial failure occured at one of the two joint interfaces with the parent material and 1 continued upward to within 1/4" of the top where a piece approximately 1" wide got blown out from the acrylic due to the compressional forces. For the $\boldsymbol{A} 3$ joints there are several cases where the failure was entirely in one of the two parent coupons and not in the joint itself. This last observation leads to the conclusion that the $A 2$ and $A 3$ bonds are approacting the flexural strength of the parent material (i.e. about 16,000 psi maximum flexural strength).

The A4 (overfilled, routed, sanded and polished) samples were significantly lower in strength than the $A 2$ and $A 3$ samples. This lower strength has no obvious explanation. Examination of the broken test samples reveals that the failure modes are very similar to those seen in the $A 2$ and $A 3$ samples. One possible interpretation for this lower strength is that the heat associated with polishing results in higher residual stresses in the surface of the acrylic than routing or sanding.

\section{$B$ Samples}

A cursory examination of the results for the $B$ samples shows that underfilling the bond joint with adhesive creates a vastly inferior bond. This 
result should not be surprising because these bonds have a substantially reduced cross sectional area. Filling the bond joint after the initial gluing to correct the problem followed by sanding and polishing clearly increases the bond strength substantially, but does not achieve the same level achieved by making the bond correctly to begin with.

A comparison of the $A 1$ and $B 1$ sample types, which are both unfinished, shows that the maximum flexural strength of the $B 1$ samples is $60 \%$ of the $A 1$ samples. This reduction in strength appears to be largely due to the reduced cross sectional area in the bond region (the joint cross sectional area of the $B 1$ samples is $\sim 75 \%$ of the acrylic cross sectional area of the $A 1$ samples). In all cases the $B 1$ samples broke close to the center of the joint which is consistent with the reduced cross sectional area of the joint and its rough surface finish.

One might expect the $B 2$ and $B 4$ samples to behave similarly because both start from the same initial underfilled bond that is finished by remove surface imperfections, albeit using slightly different approaches. For the $B 2$ samples just the bond area was finished while for the $B 4$ samples a gentle flair was introduced on both sides of the bond. The $B 2$ samples had the lowest flexural strength observed in all of the tests conducted. The $B 4$ samples followed the $A 1 / A 2$ pattern with the finished samples (B4) being better than the unfinished ones $(B 1)$. This different behavior can be explained by the observation that the $B 2$ samples all broke at one edge of the cutout region, not at the bond joint itself, while the $B 4$ samples all broke at the bond joint. The $B 2$ samples could probably have been built substantially stronger if a much larger radius had been used to blend the cutout region to the normal thickness acrylic material.

The $B 3$ samples were by fai the strongest of the $B$ samples, but were still substantially weaker (i.e. $\sim 3000$ psi) than the comparable $A$ samples ( $A 4)$ with a full cross sectional bond joint that was routed, sanded, and polished. More disturbing is the very 
wide distribution in the individual results that ranged from 5700 psi to almost 10,000 psi. Repairing an underfilled bond joint is apparently not a straight forward task and a substantial effort will need to be devoted to developing a reproducible repair technique since in the kilometer of bond joints present in the final acrylic vessel at least some will not be made correctly the first time.

\section{Samples}

In the process of casting and thermal forming acrylic panels it is difficult to maintain a close tolerance on the thickness of the material. The $C$ test samples were designed to study the bonding of two dissimilar thickness acrylic sheets. The surprising conclusion is that the variation in flexural strength is relatively independent of surface finish. Each subsample type within the $C$ series gets progressively stronger as one routs, sands, and polishes the samples, but the increase in strength is not the factors of 2 or 3 seen for other samples.

Almost all test samples broke in the same fashion, with the break beginning at the joint, continuing straight up, and then veering off away from the thicker piece. This uniform behavior almost certainly accounts for the similarity in strength between the $C$ subtypes. The $A$ and $B$ samples had a much wider variation in failure modes between subtypes as already discussed that accounts for their larger spread in flexural strength.

One other observation is that the failures occur where the flair between the joint and the thicker acrylic piece begins at the joint. This flair is substantially steeper than the one used in the B4 samples though not as severe as the discontinuity seen in the $B 2$ samples. This observation could explain why the decrease in strength from the $A$ samples to the corresponding $C$ samples (2.7) is considerable more than the ratio of the cross sectional areas (1.3). The transition from the 0.75 " acrylic to the flair is quite 
abrupt almost certainly causing a region of increased residual stress.

\section{$D$ Samples}

Misalignment of the panels to be bonded is a serious problem in the final acrylic vessel especially for the last few panels which will have to be installed in a substantially rigid structure. The $D$ samples demonstrate that careful finishing of the surface to minimize any discontinuities will ameliorate any degradation, of the flexural bond strength. This statement has been amply demonstrated by the other bond types and is a general statement that can be made about bonding two pieces of acrylic together.

The $D 1$ and $D 2$ samples have surprisingly similar maximum flexural strengths. A close of examination of the failure pattern reveals that both fail the same way with the initial break coming at one side of the joint where the discontinuity occurs. The break is very similar in shape to that of the $C$ samples. The flair is also quite steep and rough in the $C 2$ samples further supporting the general claim that any kind of discontinuity severely degrades a bond joint.

The $D 3$ and $D 4$ samples have more gentle flairs than the $D 2$ samples and the transition from the parent acrylic material to the flair is more gradual which explains their greater strength. For a mismatch of $1 / 8$ " over a thickness of 1 " the maximum flexural strength is approximately $75 \%$ of the best $A$ samples. This reduction appears reasonable in light of the reduced cross sectional area introduced by the additional sanding and polishing plus the mismatch in how the flexural loads are applied given that the piece is no longer flat.

\section{Observations and Conclusions}

Several conclusions can be made from our tests. First, any surface imperfection or discontinuity on the acrylic sheets or 
bond joint will greatly reduce the maximum flexural strength of the bond region. Second, repairing a joint is not as good as making the joint correctly in the first place. Thirdly, any misalignment or difference in thickness between panels to be bonded should have a finish that gradually blends from one side of the joint to the other. In all cases, however, the best bond is one that is well aligned, overfilled with monomer, and then finished to produce a surface free of imperfections.

\section{Acknowledgements}

We would like to thank Roger Reynolds and Herb Meyer from Reynolds Polymer Technology for all the advice they have given us concerning bonding procedures. At Los Alamos we would like to thank the staff of the Isotope and Nuclear Chemistry machine shop who made all the samples. Finally, we would like to thank David Lauben who tested the acrylic samples. This work was performed under the auspices of the US Department of Energy. 

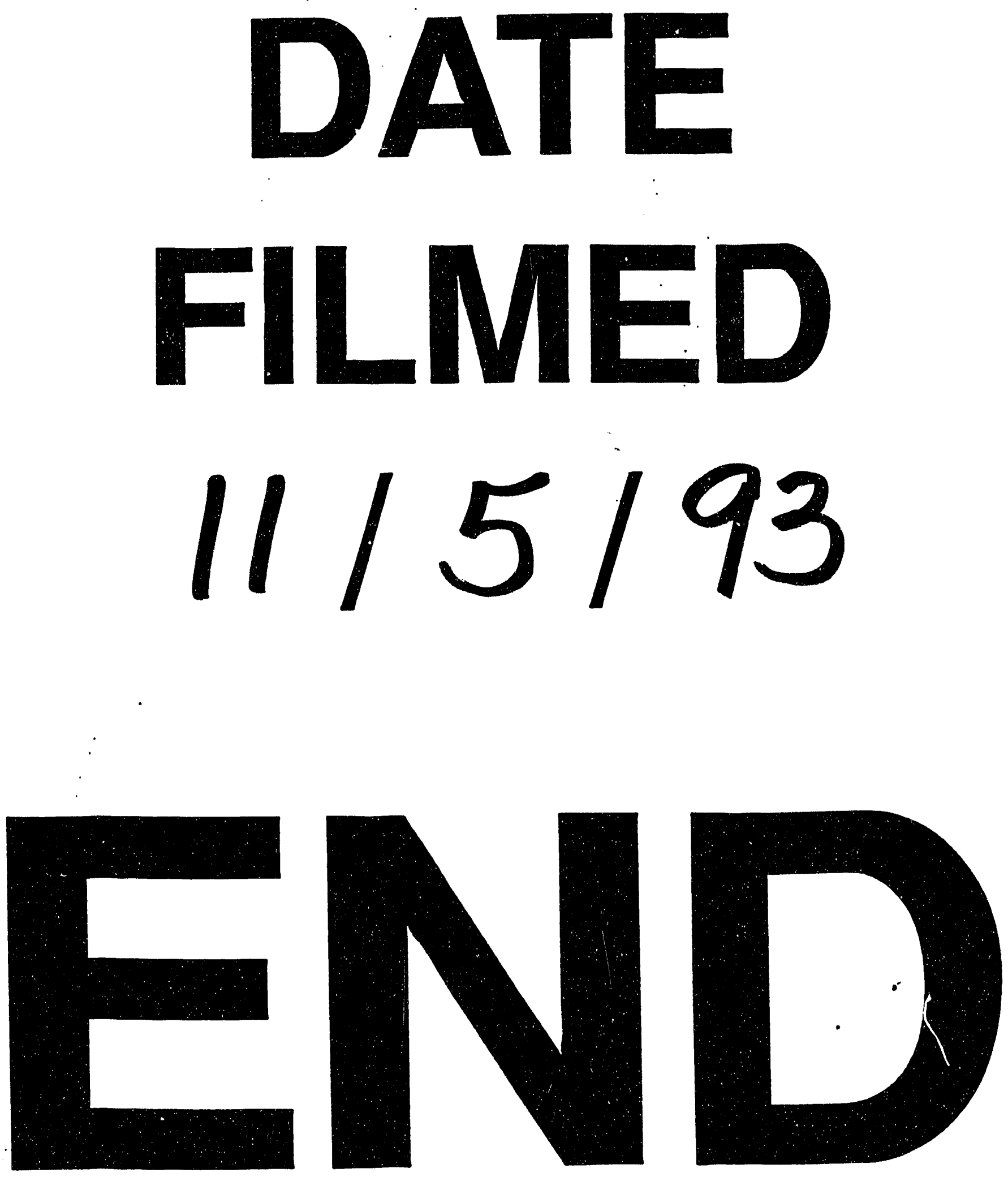
